\title{
Redes sociales como medio de promoción turística en los países iberoamericanos
}

\section{Social networks as a means of tourist promotion in the ibero-american countries}

Dr. Guillermo Antonio Gutiérrez Montoya es Decano de la Facultad de Ciencias Económicas de la Universidad Don Bosco (El
Salvador) (guillermo@udb.edu.sv) (http://orcid.org/0000-0003-1305-6030)

Dr. Miguel Ángel Sánchez Jiménez es profesor investigador del Departamento de Marketing y Comunicación de la Universidad de Cádiz (España) (miguelangel.sanchez@uca.es) (http://orcid.org/0000-0002-4271-3522)

Dra. Araceli Galiano Coronil es profesora investigadora del Departamento de Marketing y Comunicación de la Universidad de Cádiz (España) (araceli.galiano@uca.es) (http://orcid.org/0000-0003-2270-0924)

\section{Resumen}

A través de las redes sociales es posible crear una comunidad virtual alrededor de una marca, permitiendo a los usuarios que lo deseen informarse e interaccionar con las publicaciones realizadas por la misma. En este contexto destacan las redes sociales como canales para obtener y difundir información sobre experiencias de viaje, siendo de gran influencia por parte de los turistas a la hora de tomar una decisión de compra. De esta manera, es de interés conocer cómo los destinos turísticos utilizan estas redes sociales para realizar sus estrategias de marketing. Así, el objetivo del presente estudio es analizar la actividad y efectividad de las redes sociales oficiales para la promoción turística de los países iberoamericanos. Para ello se consideran las variables de "presencia», "engagement" y "tipo de publicaciones» que se miden a través de una serie de indicadores de rendimiento mediante la herramienta de análisis online Fanpage Karma ${ }^{\circledR}$. Los resultados especifican que cada red social puede ser utilizada para un objetivo en particular. Facebook destaca como la principal red social más completa para la promoción del destino turístico con un mayor número de seguidores. Instagram se convierte en la red social principal para general una mayor interacción con los usuarios mientras Twitter va evolucionando a un canal de noticias y comunicación. Destacar también la importancia del carácter visual de las publicaciones para el ámbito turístico, siendo las que más interacciones suelen generar.

\begin{abstract}
Through social networks it is possible to create a virtual community around a brand allowing users who wish to inform themselves and interact with the publications made by the brand itself. In this context, these social networks stand out as a channel to obtain and disseminate information on travel experiences, being of great influence on the part of tourists when making a purchase decision. In this way, it is interesting to know how tourist destinations use these social networks to carry out their marketing strategies. Thus, the objective of this study is to analyze the activity and effectiveness of official social networks for tourism promotion in Ibero-American countries. To do this, we consider the variables of "presence", "engagement" and "type of publications" that are measured through a series of performance indicators using the online analysis tool Fanpage Karma ${ }^{\circledR}$. The results specify that each social network can be used for a particular goal. Facebook stands out as the most complete social network for the promotion of tourist destination with a greater number of followers. Instagram becomes the main social network for general greater interaction with users while Twitter evolves into a news and communication channel. Also highlight the importance of the visual nature of publications for the tourism sector, being those that most often generate interactions of the social networks studied are useful according to the objective to be achieved.
\end{abstract}

\section{Palabras claves I keywords}

Turismo, marketing, redes sociales, ROI, engagement, países iberoamericanos.

Tourism, marketing, social networks, ROI, engagement, Ibero-American countries.

Forma sugerida de citar: Gutiérrez Montoya, G. A., Sánchez Jiménez, M. Á., \& Galiano Coronil, A. (2018). Redes sociales como medio de promoción turística en los países iberoamericanos. Retos Revista de Ciencias de la Administración y Economía, 15(8), 135-150. https://doi.org/10.17163/ret.n15.2018.09 


\section{Introducción}

Una comunidad virtual alrededor de una marca es un grupo especializado construido sobre un conjunto estructurado de relaciones sociales entre admiradores de productos o servicios de una marca específica (Pathak, \& Pathak-Shelat, 2017). La razón por el cual las marcas establecen una comunidad virtual alrededor de ellas puede explicarse por su deseo de fortalecer la construcción de marca a través de la retroalimentación que ofrece la relación establecida con los consumidores (Gummerus et al., 2012). Una comunidad de marca exitosa puede ayudar a fortalecer la lealtad de marca de los consumidores y servir como un canal para la comunicación y el intercambio de conocimiento entre las marcas y los consumidores, así como entre los propios consumidores (Bruhn et al., 2014). Además, la investigación empírica ha demostrado que los consumidores que participan en comunidades de marcas tienden a tener una relación más profunda y más estrecha con la marca que aquellos que no lo son (v.gr. Laroche et al., 2013; Potgieter \& Naidoo, 2017; Rosenthal \& Brito, 2017). Por lo tanto, las comunidades de marcas en línea funcionan no solo como un canal para que las entidades se comuniquen con los clientes, sino también como una herramienta importante para construir relaciones con los clientes.

En la actualidad, un gran número de personas dispuestas a viajar a un destino turístico eligen las alternativas de comunicación y planificación en línea, entre las que destacan las redes sociales (Dina \& Sabou, 2012). Así, la oferta turística opta por adaptarse a la nueva tendencia de comportamiento de los turistas, volviéndose cada vez más activas en las redes sociales, que han revolucionado las interacciones comunicativas y, en consecuencia, la comercialización de destinos turísticos y estrategias empresariales (Sotiriadis \& van Zyl, 2013).

La medición del valor de un negocio desde las Tecnologías de la Información y la Comunicación ha sido tradicionalmente problemática, lo que se extiende a los medios sociales (Stockdale et al., 2012; Geho et al., 2011). Los medios sociales son diferentes a los medios tradicionales, por lo tanto, requieren un enfoque diferente para su medición. Si bien el impacto de la actividad online se puede medir utilizando métricas cuantitativas definidas, las redes sociales generan una cantidad considerable de datos cualitativos que las métricas tradicionales, por sí solas, no pueden abordar o cuantificar en términos monetarios (Fisher, 2009).

De esta manera, el objetivo del presente estudio es analizar la actividad y efectividad de las redes sociales oficiales para la promoción turística de los países iberoamericanos a través de la medición de una serie de indicadores de rendimiento mediante la herramienta de análisis online Fanpage Karma ${ }^{\circledR}$. En este caso la muestra de países iberoamericanos seleccionados para el estudio son los 22 miembros de la Secretaría General Iberoamericana: Andorra, Argentina, Bolivia, Brasil, Chile, Colombia, Costa Rica, Cuba, Ecuador, El Salvador, España, Guatemala, Honduras, México, Nicaragua, Panamá, Paraguay, Perú, Portugal, República Dominicana, Uruguay y Venezuela.

1 Disponible en línea en: http://segib.org/ 


\section{Marco teórico}

\subsection{Importancia del sector turístico a nivel mundial}

El turismo es la industria de servicios más grande del mundo, considerándose uno de los sectores de mayor importancia en muchos países y uno de los principales motores de la economía mundial, cuya importancia es cada vez mayor (Devi, 2015), siendo en la actualidad uno de los cinco sectores que proporcionan más ingresos en el $83 \%$ de los países y la principal fuente de moneda extranjera en el 38\% de los países en el año 2016 (OMT, 2017).

Según el estudio realizado por World Travel \& Tourism Council (WTTC, 2017), el turismo es un sector clave para el desarrollo económico y la creación de empleo en todo el mundo. Respecto al desarrollo económico, en 2016 el turismo contribuyó directamente con 2,3 billones de dólares a la economía mundial, lo que equivale al $10,2 \%$ del PIB mundial. Esta contribución al PIB creció un 3,1\% en 2016, por encima del conjunto de la economía mundial, que creció un 2,5\%. Además de superar el crecimiento económico mundial, el sector turístico también rebasó a otros sectores económicos globales importantes en 2016. Específicamente, el crecimiento directo del PIB referido al turismo fue más fuerte que el crecimiento registrado en los servicios financieros y empresariales, la distribución y el transporte, y solo fue ligeramente menor que el crecimiento del sector de las comunicaciones.

En lo que respecta a la creación de empleo, el estudio de WTTC (2017) especifica que en 2016 el turismo contribuyó directamente a la creación de 109 millones de empleos, aproximadamente 1 de cada 10 en todo el mundo. La contribución directa del turismo al empleo creció un $1,8 \%$ en 2016 con respecto al año anterior, lo que significa que casi 2 millones de empleos netos adicionales fueron generados directamente por el sector y un total de alrededor de 6 millones de nuevos empleos creados como resultado de la actividad.

Según el precitado estudio de WTTC (2017), las perspectivas de futuro son también positivas. En el año 2017 se prevé que la contribución total del turismo a la economía mundial crezca un 3,5\%. Se espera un sólido crecimiento continuo a escala mundial entre los principales indicadores económicos de la contribución al PIB, la creación de empleo, la inversión y las exportaciones. Un desafío clave para el turismo en 2017 será una desaceleración general en el poder de consumo que afectará al gasto, debido a la combinación de una inflación más alta causada por la recuperación de los precios del petróleo -que podría tener impactos negativos para las tarifas aéreas-, el aumento del coste de la deuda a medida que aumentan los tipos de interés y una desaceleración de la creación de empleo en todo el mundo. La previsión para los próximos diez años es aún mejor, pues se espera que la contribución directa de turismo al PIB crezca un promedio de 3,9\% y genere más de 380 millones de empleos en todo el mundo.

\subsection{Las Tecnologías de la Información y Comunicación (TIC) y los me- dios sociales en el sector turístico}

Las TIC han cambiado la manera en que se informa sobre el turismo y la forma en que las compañías y comunidades turísticas trabajan en el diseño y la comer- 
cialización de sus productos, del mismo modo en que se ha transformado la forma en que los turistas planifican, reservan y viven sus experiencias turísticas (Munar, 2012).

Ferrá \& Cardona (2015) consideran que las TIC forman parte de la cultura actual y amplían las posibilidades de desarrollo social personal. Suele incluirse en el concepto TIC no solamente la informática y sus tecnologías asociadas, telemática y multimedia, sino también los medios de comunicación de todo tipo: los medios de comunicación social y los medios de comunicación interpersonales tradicionales con soporte tecnológico como el teléfono.

Según Xiang \& Gretzel (2010), la web 2.0 ha sido el principio de dos tendencias fundamentales: la aparición de los medios sociales y el aumento en la cantidad de información que se puede encontrar. Estos son dos elementos que se encuentran estrechamente vinculados, ya que el aumento de la información y los elementos turísticos en la red se han encontrado con la información producida por los usuarios como consecuencia del surgimiento de la web 2.0 y de los nuevos elementos sociales relacionados con este avance (Sotiriadis \& van Zyl, 2013); y que se combinan con los datos y contenidos extras que tienen origen en las compañías y empresas encargadas de gestionar los destinos turísticos (Hudson \& Thal, 2013).

Ante este panorama, la información que se comparte en los medios sociales se reconoce como un punto fundamental de información que puede facilitar la programación de los viajes o incluso puede influenciar en la toma de decisiones de un potencial turista (Kang \& Schuett, 2013). Dichos turistas potenciales suelen verse influenciados por las experiencias y opiniones de terceros a la hora de tomar decisiones en lo que respecta a su viaje (Zeng \& Gerritsen, 2014). Por razones como las expuestas en precedencia, la web 2.0 y los medios sociales han transformado la manera en que los usuarios programan y compran sus viajes. De esta forma, la web 2.0 ha quitado cierta importancia a la web de antaño, ya que la información, hablando desde un punto de vista cuantitativo y cualitativo, ha mejorado considerablemente, debido a que los propios usuarios amplían dicha información con sus propias experiencias.

Las características propias del sector turístico, en general, y del sector hotelero, en particular, hacen que sea mayor su repercusión en los medios sociales. El sector turístico, como cualquier sector de servicios, vende productos intangibles que se caracterizan por ser inseparables, perecederos y heterogéneos, de ahí que el proceso de comunicación sea un elemento vital en la oferta (Sirakaya \& Woodside, 2005). Por esta razón, el uso de los medios sociales ha permitido nuevas posibilidades de comunicación y ha proporcionado un impacto sustancial en la demanda y la oferta turística (Martínez et al., 2013).

\subsection{Medición del ROI en las redes sociales}

Aunque se puede argumentar que solo las ganancias financieras pueden ser medidas en valor monetario, las ganancias no financieras de los medios sociales pueden ser igualmente beneficiosas ya que otorgan una rentabilidad intangible, como una mejor comunicación con los clientes, la conciencia de marca, entre otras. Sin embargo, aunque estas consideraciones pueden ser ventajosas para cualquier negocio, medir 
tales beneficios intangibles es problemático. Mientras, algunas métricas, como «el aumento de seguidores en Facebook o Twitter» no se refieren necesariamente a un aumento de las ventas, es claro ver que la presencia de una marca a través de las plataformas de medios sociales, en última instancia, puede conducir a la ganancia financiera (Blanchard, 2012).

El Return On Investment (ROI), o retorno de la inversión, por sus siglas en inglés, simplemente se refiere a la idea de que algo de valor ha resultado de una inversión de tiempo, energía o dinero. En los círculos financieros, el ROI suele reducirse a una fórmula (Bragg, 2012), que se calcula simplemente restando el costo de una inversión de los ingresos recibidos de la inversión, dividido por el mismo costo de inversión. Es normal que las marcas busquen ganancias, pero los medios sociales no pueden interpretarse únicamente como una medida de tales beneficios y por esta razón es importante estar familiarizado con otras formas de utilización del ROI. En primer lugar, si una institución está presente en los medios sociales, es porque ya hay alguien hablando sobre ello $\mathrm{y}$, en segundo lugar, estos medios no ofrecen nada más que nuevas maneras de interactuar y comunicarse con las audiencias y clientes prospectivos. Evitarlos es como negarse a tener un teléfono o una oficina de atención al cliente. Además, la necesidad de comparar el Social Media Marketing con la publicidad más tradicional nos hace perder de vista el aspecto más importante de la comunicación 2.0, que es su bidireccionalidad (Schmidt, 2014).

No existen unas normas que definan de forma clara y fácil cómo se obtienen los datos, cuáles son las métricas más adecuadas para obtener la información (Leeflang et al., 2014). Cuando se aborda la medición de la rentabilidad en medios sociales se contempla un beneficio en valor, el llamado IOR (Impact On Relationship) o Retorno en Relaciones. El IOR se considera como el valor acumulado que otorga una persona a una marca, debido al cultivo de esta relación. El IOR es un valor y se puede medir su impacto a través de recomendaciones, participación o lealtad. Los medios sociales son fácilmente rentables para conseguir beneficios no económicos, de ahí que se hable también del SROI o ROI Social (Taladriz-Mas, 2013).

Una vez que el foco de retorno de la inversión con respecto a los medios sociales se ha ampliado, las marcas pueden empezar a considerar el «impacto de las relaciones» o IOR. Este enfoque se centra en el análisis de las interacciones de medios sociales entre una marca y sus fans/seguidores y luego determinar cómo esa relación puede ser fructífera (Geho \& Dangelo, 2012).

Para medir este impacto de la relación se necesita la creación de un conjunto de indicadores y métricas únicas, que midan el impacto en los usuarios y la rentabilidad de la marca en la web social y sirva también como análisis competitivo (González Fernández-Villavicencio, 2016).

Los indicadores clave de desempeño (KPIs, Key Performance Indicator) son métricas utilizadas para cuantificar objetivos que reflejan el rendimiento de una organización. El acto de monitorizar los indicadores clave de desempeño en tiempo real se conoce como «monitorización de actividad de negocio». Los KPIs son vehículos de comunicación y suelen estar atados a la estrategia de la organización. Por su parte Castelló (2012) comenta que un KPI es un indicador clave de rendimiento, una 
variable que está ligada a un objetivo en los medios sociales y permite monitorizar el estado de avance o grado de cumplimiento del objetivo. Los KPIs sirven para saber cómo dinamizar los canales de la marca en redes sociales ya que los resultados darán pistas sobre cómo seguir aplicando la estrategia.

\section{Metodología}

\subsection{Diseño de investigación}

Para llevar a cabo el análisis se ha tomado como base el modelo PRGS (Presencia, Respuesta, Generación y Sugerencia), propuesto por IAB Spain Research para elaborar su IV Estudio de la Actividad de las marcas en Medios Sociales en los años 2012-2016. Este modelo ha sido utilizado en diversos estudios como, por ejemplo, los realizados por Sánchez \& Giraldo (2015), Bueno et al. (2017) y Giraldo \& Martínez (2017).

Este modelo se fundamenta en 4 variables (Presencia, Respuesta, Generación y Sugerencia) para medir la actividad y la efectividad de las marcas en redes sociales (Giraldo \& Martínez, 2017):

- $\quad$ P (Presencia): viene dada por el número de fans o seguidores y el número de publicaciones que la marca realiza en la página. $\mathrm{P}=\mathrm{n}^{\circ}$ de seguidores y $\mathrm{P}=\mathrm{n}^{\circ}$ de post.

- $\quad \mathrm{R}$ (Respuesta): viene dada por el número de me gusta de los usuarios que los mismos generan ante las publicaciones de la marca. $\mathrm{R}=$ likes o me gusta $/ \mathrm{n}^{\circ}$ de fans.

- G (Generación): viene dada por el número de comentarios de los usuarios que los mismos generan ante las publicaciones de la marca. $\mathrm{G}=$ comentarios $/ \mathrm{n}^{\circ}$ de fans.

- S (Sugerencia) viene dada por el número de comparticiones o recomendaciones de los usuarios que los mismos generan ante las publicaciones de la marca. $\mathbf{S}=$ Compartir/ $\mathrm{n}^{\mathrm{o}}$ de fans.

Para el presente estudio, las variables de «respuesta», "generación» y «sugerencia» se han unido en una sola variable denominada "engagement», que corresponde a la suma de las tres anteriores. La formulación y el cálculo del «engagement» ha sido tratada en varios estudios como los de Castelló (2012), Oviedo-García et al. (2014) y Buhalis \& Mamalakis (2015).

Además, como aportación original al modelo, se ha considerado de interés para analizar la actividad añadir la variable «tipo de publicaciones», a través de la cual se obtiene información acerca del tipo de publicaciones de las redes sociales oficiales de los países iberoamericanos. De esta manera, la modificación del modelo de evaluación de la presente investigación toma en consideración las variables de «presencia» proveniente del modelo PGRS, «engagement» $\mathrm{y}$ «tipo de publicaciones».

Los KPIs utilizados en cada variable para cuantificar los resultados han sido los siguientes (tabla 1): 
Tabla 1. Variables e indicadores de rendimiento utilizados en el análisis junto con su descripción

\begin{tabular}{|l|l|l|}
\hline \multicolumn{1}{|c|}{ Variable } & \multicolumn{1}{|c|}{ Indicadores } & \multicolumn{1}{c|}{ Descripción } \\
\hline Presencia & Número de seguidores & $\begin{array}{l}\text { Establece el número de seguidores de la red so- } \\
\text { cial del hotel }\end{array}$ \\
\cline { 2 - 3 } & $\begin{array}{l}\text { Número de publicaciones } \\
\text { y publicaciones/día }\end{array}$ & $\begin{array}{l}\text { Índica cuantas publicaciones ha realizado el } \\
\text { hotel }\end{array}$ \\
\hline $\begin{array}{l}\text { Engagement o } \\
\text { actividad }\end{array}$ & $\begin{array}{l}\text { Número total de interac- } \\
\text { ciones / número de publi- } \\
\text { caciones }\end{array}$ & $\begin{array}{l}\text { Índica cuál es el número total de las interac- } \\
\text { ciones de los usuarios por publicación de la } \\
\text { gusta, me encanta, sorprendido, risa, etc.), los } \\
\text { comentarios y las veces que se ha compartido } \\
\text { entre el número de publicaciones }\end{array}$ \\
\hline Publicaciones & Tipo de publicaciones & $\begin{array}{l}\text { Indica que tipo de publicaciones se ha realiza- } \\
\text { do, bien en enlaces, videos o imágenes }\end{array}$ \\
\hline
\end{tabular}

\subsection{Recopilación de los datos}

Para la medición de los indicadores de las redes sociales oficiales de los 22 países iberoamericanos, se ha empleado la herramienta online Fanpage Karma® que permite el análisis y la monitorización de medios sociales. Esta herramienta ha sido utilizada en varios estudios de investigación para el análisis de redes sociales entre los que destacan (v.gr) los de Jayasingh \& Venkatesh (2015), Huertas \& Marine-Roig (2016), MartínezFernández et al. (2015) y Márquez-Domínguez et al. (2017). Además, también ha sido utilizado para la realización de estudios turísticos como los de Wozniak et al. (2017) y Huertas et al. (2015).

En este caso se han seleccionado como objeto de análisis las redes sociales oficiales de promoción turística utilizadas por los 22 países iberoamericanos por considerarse los más relevantes para para estudiar el turismo de la zona. De esta manera, se ha obtenido una base de datos con las redes sociales oficiales de turismo que utilizan estos 22 países con su enlace correspondiente. Se han tenido en cuenta las redes sociales de Facebook, Twitter, Instagram y Youtube por considerarse las más destacadas en el sector turístico (Beezhotels, 2016). La obtención de los datos se realizó para un periodo de tiempo de seis meses, correspondientes desde el día 1 de mayo de 2017 hasta el 31 de octubre de 2017.

\section{Resultados}

A continuación, se exponen los resultados obtenidos en el análisis de las redes sociales de los 22 países iberoamericanos en el periodo de seis meses señalados, teniendo en cuenta las variables ya comentadas de presencia, engagement y tipo de publicaciones. En los apartados 5.1, 5.2 y 5.3 se especifican los resultados obtenidos por 
cada una de las redes sociales y en el 5.4 los resultados comparativos entre los países iberoamericanos.

\subsection{Presencia}

El estudio para comprobar la presencia de los países iberoamericanos en las redes sociales oficiales para su promoción turística se ha dividido en dos apartados, teniendo en cuenta los siguientes indicadores: primero se analiza la media de número de seguidores en cada red social y, posteriormente, la media del número de publicaciones. Para ello se tienen en cuenta las redes sociales de Facebook, Twitter, Instagram y Youtube.

Antes de comenzar el análisis se ha comprobado que todos los países iberoamericanos tienen un perfil abierto en cada una de las redes sociales que se han tomado como referencia en el estudio y se ha realizado una base de datos con los resultados teniendo en cuenta los indicadores mencionados anteriormente.

En lo que concierne a los resultados en la media de número de seguidores por cada red social puede comprobarse que destaca con mucha diferencia Facebook con el 67,2\% del total, le sigue Twitter con el 20,9\% y, a más distancia, se encuentran Instagram y Youtube con el 10,7\% y el 1,1\% respectivamente (figura 1).

Figura 1. Media de número de seguidores por red social

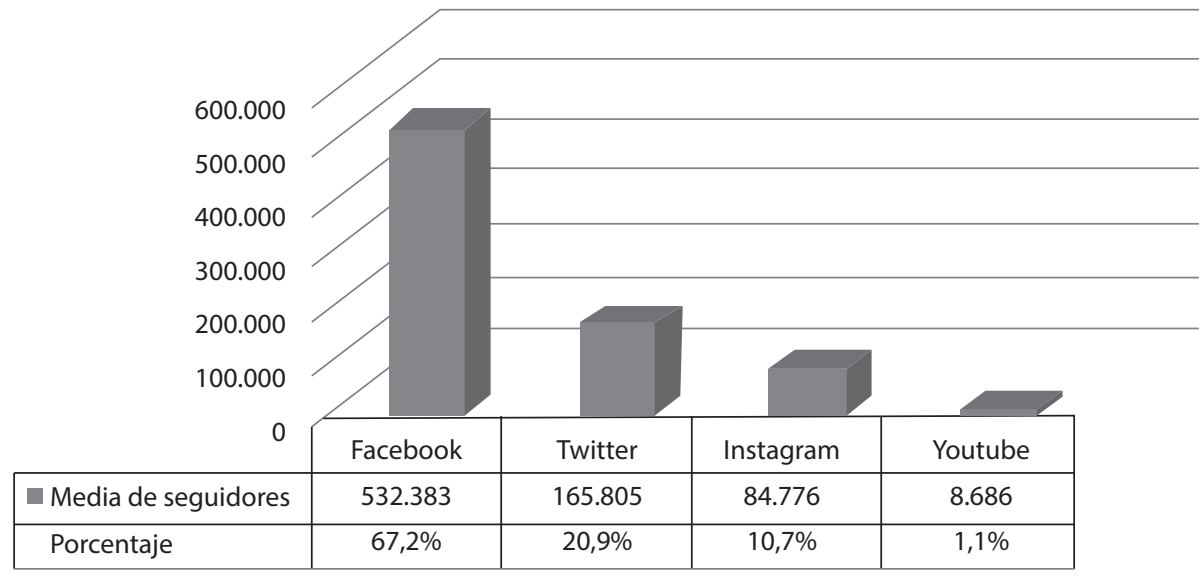

En lo que respecta a la media del número de publicaciones, Twitter es la red social que más destaca con el 55,1\% del total, seguida de cerca por Facebook con el $32,1 \%$. A bastante más diferencia se encuentra Instagram con el 10,4\% y por último Youtube con el 2,4\% (figura 2). 
Figura 2. Media de número de publicaciones por red social

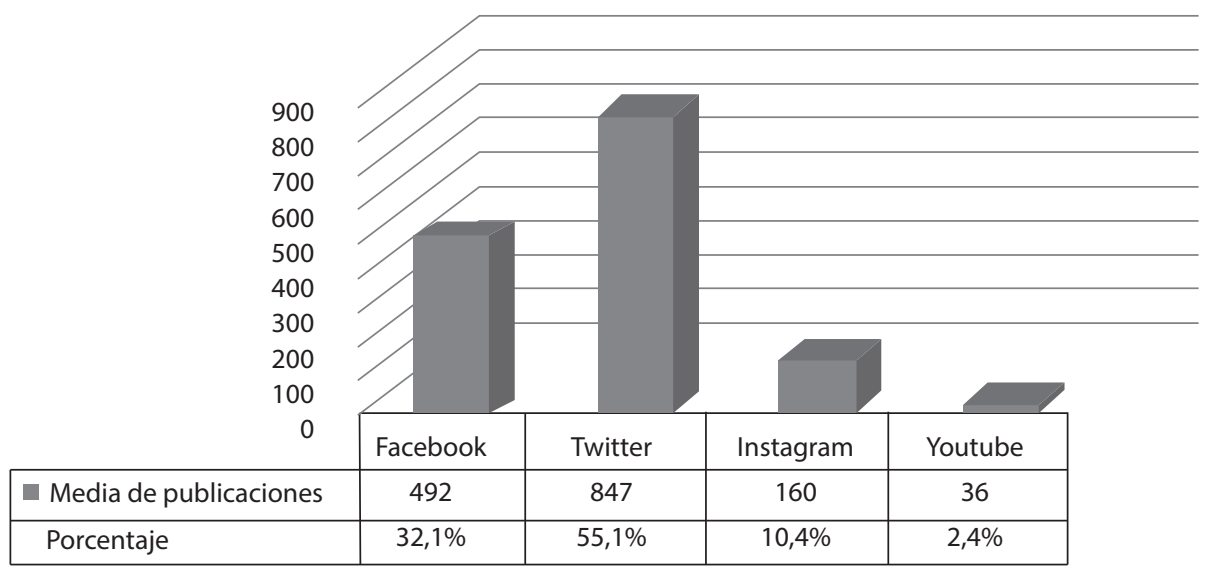

\subsection{Engagement}

Una vez se ha comprobado la presencia de los países iberoamericanos en las redes sociales oficiales, resulta de especial interés analizar cuál es la efectividad de su utilización medida a través de la variable engagement.

Para calcular el engagement de cada perfil de redes sociales de los países iberoamericanos se ha tenido en cuenta la siguiente fórmula:

$$
E=\frac{n i(n r+n c+n c o)}{n p}
$$

$E=$ Engagement .

$n i=$ Número de interacciones.

$n r=$ Número de reacciones.

$n c=$ Número de comentarios.

nco $=$ Número de compartidos.

$n p=$ Número de publicaciones

En la figura 3 puede verificarse que las dos redes sociales con mayor engagement con mucha diferencia respecto a las demás son Instagram y Facebook. En este caso destaca Instagram con el $67,2 \%$ del total y Facebook con el 30,4\%. Se considera, por tanto, que los usuarios participan e interaccionan mucho más con estas dos redes sociales. Llama la atención el caso de Instagram que pese a no tener un gran número de seguidores ni publicaciones sea la red social donde se genera mayor engagement. 
Figura 3. Media de engagement por red social

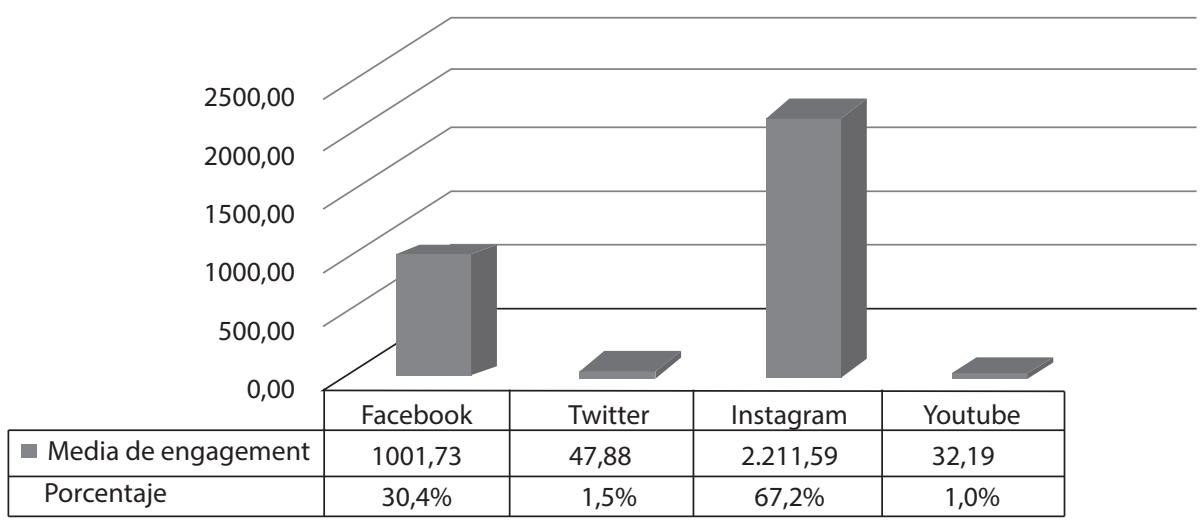

\subsection{Tipo de publicaciones}

En este apartado se ha analizado el tipo de publicaciones realizadas por los diferentes perfiles de redes sociales de los países iberoamericanos. Se verifica, por tanto, la media de tipo de publicaciones por red social llevadas a cabo durante los seis meses objeto de estudio.

En la figura 4 se comprueba la media de las publicaciones en Facebook por cada tipo de publicación. Destacan los mensajes o post de imágenes, siendo el $54,6 \%$ del total, mostrando los destinos turísticos una apuesta manifiesta por realizar publicaciones de carácter visual. Le sigue, a mayor distancia, tantos los mensajes de enlace como los de videos con el $24,8 \%$ y el $20,6 \%$ del total respectivamente.

Figura 4. Media de tipos de publicaciones en Facebook

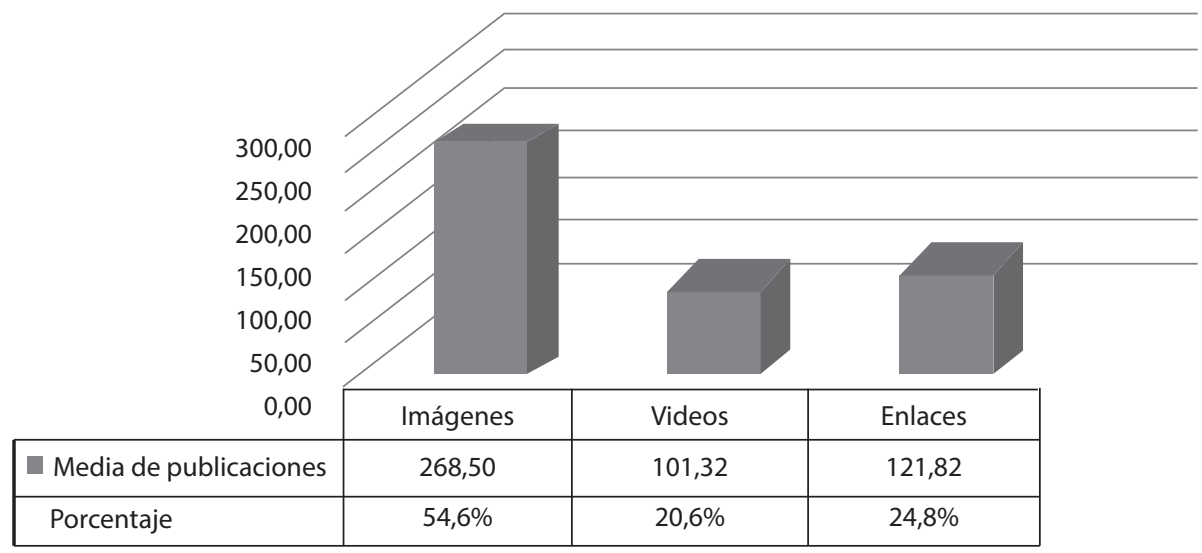


En lo que respecta a Twitter (figura 5), se contrasta que la mayoría de los mensajes o tuits a lo largo de los seis meses van referidos a imágenes con un 42,4\% del total. Las demás publicaciones están muy igualadas, siendo las de solo texto del 21,2\%, las de imágenes y enlaces del 19,2\% y las de enlaces del 17,3\% del total. Puede comprobarse en el caso de Twitter que el contenido está más repartido que en Facebook, destacando la importancia de las imágenes pese a no ser una red social propicia para ello.

Figura 5. Media de tipos de publicaciones en Twitter

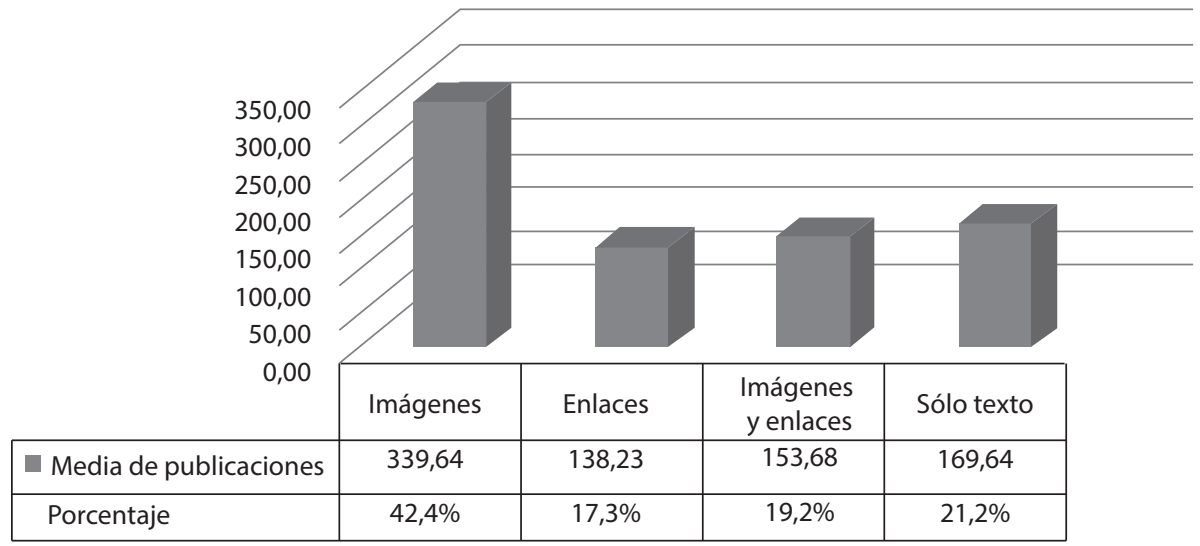

La red social Instagram solo permite la publicación de contenido mediante imágenes y videos. En este caso puede comprobarse en la figura 6 que el $92 \%$ de las publicaciones son imágenes mientras que el $8 \%$ son videos (figura 6).

Figura 6. Media de tipos de publicaciones en Instagram

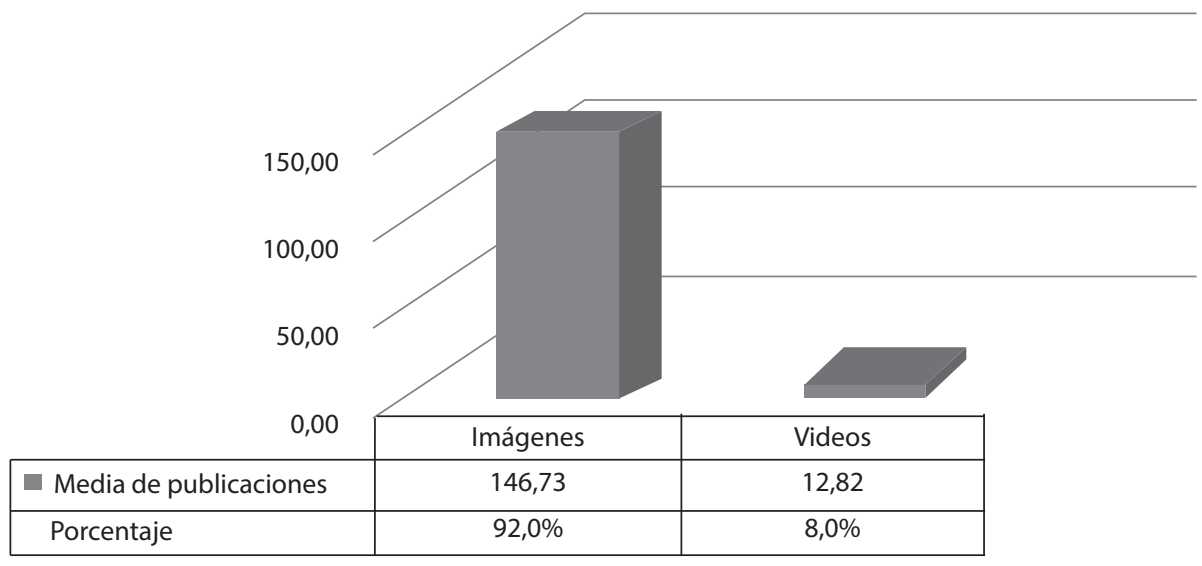


Además, en Facebook se conocen las interacciones obtenidas por cada tipo de publicación (figura 7). Puede comprobarse que hay una gran similitud en las interacciones producidas en cada tipo de publicación, destaca por poco margen los enlaces con el $42,1 \%$ del total, y posteriormente los videos y las imágenes con un $29,9 \%$ y un $28 \%$ del total respectivamente.

Figura 7. Media de interacción de las publicaciones en Facebook

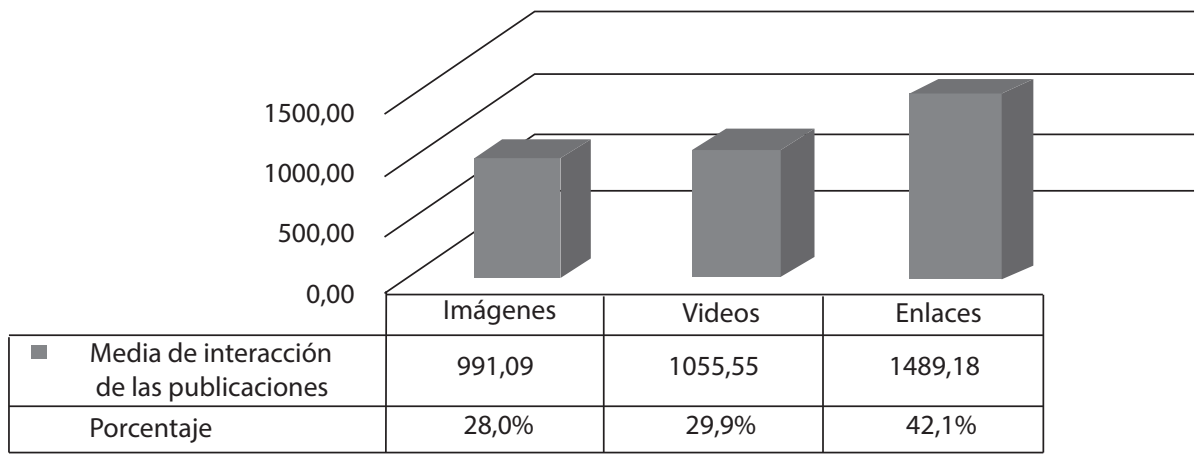

\subsection{Resultados comparativos entre los países iberoamericanos}

En este apartado se comentarán los resultados principales referidos a los países iberoamericanos. En Facebook, en general, se observa una gran cantidad de seguidores y una muy buena participación de los usuarios. Respecto al número de seguidores destacan los países de México, Portugal, Ecuador y Argentina, situándose por encima del millón de seguidores. Respecto el número de publicaciones destacan con diferencia los países de Bolivia, Chile y Honduras con más de siete publicaciones diarias durante el periodo de estudio. Sobre el engagement o las interacciones por cada publicación destaca sobre todo Costa Rica situándose por encima de las 9000 interacciones por cada publicación, le siguen con más de 2500 interacciones por cada publicación los países de México y República Dominicana.

Twitter destaca por ser la red social con mayor número de publicación en este estudio, no obstante, se encuentra por debajo de Facebook en seguidores y sobre todo en el engagement. Respecto al número de seguidores destaca con bastante diferencia México con más de un millón y medio. Le siguen con mayor número de seguidores Venezuela y Ecuador por encima de los 300000 seguidores. En publicaciones destacan los países de Portugal, España, Venezuela y Paraguay con más de nueve publicaciones al día, destacando también Nicaragua y Chile con más de cuatro publicaciones al día. Respecto a las interacciones destaca especialmente México con más de 400 interacciones de publicación y España con 237, a bastante distancia con más de 60 interacciones por publicación se encuentran Argentina, Costa Rica y Perú.

En lo que concierne a Instagram, como se ha comprobado anteriormente, el número de seguidores y de publicaciones son bastante inferiores a Facebook y Twitter, no 
obstante, destaca sobre todo por el gran resultado alcanzado en el nivel de engagement situándose por delante de las demás redes sociales. En el número de seguidores destacan los países de España, Paraguay, Chile y Portugal por encima de los 200 000. En el número de publicaciones sigue destacando Chile, pero seguida muy de cerca por El Salvador, México, España, Portugal y Perú, todas por encima de 1,4 publicaciones al día. En las interacciones destaca España con más de 9000 interacciones por publicación, seguidas con más de 6000 seguidores los países de Portugal, Costa Rica, Perú y Chile.

Como se ha evidenciado con meridiana claridad ut supra, en Youtube los resultados de presencia y engagement son bastante inferiores que en las demás redes sociales. No obstante, hay algunas provincias que sí han tenido una participación activa subiendo más de cien videos en el periodo de estudio como son Nicaragua, Uruguay y Venezuela. En las interacciones destaca por encima de los demás Ecuador con más de 300 interacciones por video subido, seguida a bastante distancia por Portugal y México con más de 50.

\section{Conclusiones}

En el estudio se ha podido comprobar, en general, el gran interés que tienen los países en promocionarse como destino turístico a través de las diferentes redes sociales. Todos los países iberoamericanos intervienen en las redes sociales y todos lo hacen, en mayor o menor medida, de forma activa.

Destaca Facebook como la red social principal para la promoción de los destinos turísticos de los países objeto de estudio, siendo la red social con mayor número de seguidores y la segunda red social en número de publicaciones y engagement. Respecto Twitter e Instagram se ha comprobado que cada una puede utilizarse con objetivos distintos. Twitter destaca por ser la red social con mayor número de publicaciones y la segunda, a bastante distancia de Facebook, en número de seguidores, no obstante, tiene un nivel muy bajo en el engagement o interacciones, por lo tanto, la convierte en una red social más de carácter informativo. Al contrario sucede con Instagram que pese a estar bastante por debajo de Facebook y Twitter respecto al número de seguidores y publicaciones es la red social donde se produce una mayor interacción en sus publicaciones estableciéndose como una plataforma ideal para generar compromiso o engagement entre los usuarios en la información turística. Por último, aunque la actividad y el engagement en Youtube no es alto, hay varios países que han subido bastantes videos en el periodo de estudio permitiendo dar a conocer visualmente su país como destino turístico.

En lo que respecta a los tipos de publicaciones destacan con diferencia el contenido de imágenes, siendo la más utilizada en todas las redes sociales analizadas -excepto Youtube que solo permite videos-. No obstante, en el análisis de la interacción por cada tipo de publicación se comprueba que hay mucha igualdad, no habiendo ningún tipo de publicación que destaque por encima de otra. Sobre los videos destacar que aunque la actividad de los videos en Youtube y en las demás redes social no es el más alto sí permite llamar la atención y conseguir una alta interacción permitiendo dar a conocer visualmente los destinos turísticos, por lo que se considera un tipo de contenido interesante de mostrar a los usuarios. 
Respecto a las consideraciones empresariales de los resultados para los destinos turísticos se recomienda el establecimiento de un objetivo particular para cada red social. Resulta esencial una intervención activa y frecuente tanto en Facebook e Instagram, realizando una buena estrategia de marketing de contenido ya que son las redes sociales que generan más interacción con bastante diferencia, dejando Twitter como canal de comunicación e información. También se recomienda un mayor interés en las publicaciones de carácter visual ya que son las imágenes y videos las que suelen generar mayores interacciones, esto también se denota en el aumento de importancia que está teniendo la red social de Instagram. En este mismo sentido, aunque Youtube sea un canal con escasa interacción sí consta de una buena cantidad de reproducciones de video que pueden dar a conocer mejor el destino turístico por lo que se recomiendo crear nuevo contenido a lo largo del tiempo.

\section{Referencias}

Beezhotels (2017). “Top 5 de redes sociales para hoteles”. (https://goo.gl/QZNkjE) (27-07-2017). Blanchard, O. (2012). El retorno de la inversión en Social Media. Anaya Multimedia.

Bragg, S. M. (2012). Business ratios and formulas: a comprehensive guide. London: John Wiley \& Sons.

Bruhn, M., Schnebelen, S., \& Schäfer, D. (2014). Antecedents and consequences of the quality of e-customer-to-customer interactions in B2B brand communities. Industrial Marketing Management, 43(1), 164-176. https://doi.org/10.1016/j.indmarman.2013.08.008

Bueno, M. Á. B., Meroño, M. C. P., \& Piernas, J. M. P. (2017). Las redes sociales aplicadas al sector hotelero. International journal of scientific management and tourism, 3(2), 131-153.

Buhalis, D., \& Mamalakis, E. (2015). Social media return on investment and performance evaluation in the hotel industry context. Information and Communication Technologies in Tourism 2015, 241-253. https://doi.org/10.1007/978-3-319-14343-9_18

Castelló Martínez, A. (2012). Del ROI al IOR: el retorno de la inversión de la comunicación empresarial y publicitaria en medios sociales. Introducción a la Investigación de Medios Publicitarios. Alicante: Universidad de Alicante.

Devi, S. V. (2015). A study on tourist satisfaction and their preference to act as responsible tourist for sustainable tourism. International Journal on Global Business Management \& Research, 3(2), 65-71.

Dina, R., \& Sabou, G. (2012). Influence of social media in choice of touristic destination. Cactus Tourism Journal, 3(2), 24-30.

Ferrá, F. C. M., \& Cardona, J. R. (2015). Presencia de las cadenas hoteleras españolas en los medios sociales. CULTUR-Revista de Cultura e Turismo, 9(1), 05-35.

Fisher, T. (2009). ROI in social media: A look at the arguments. Journal of Database Marketing \& Customer Strategy Management, 16(3), 189-195. https://doi.org/10.1057/dbm.2009.16

Geho, P. R., \& Dangelo, J. (2012). The evolution of social media as a marketing tool for entrepreneurs. The Entrepreneurial Executive, 17, 61.

Geho, P. R., Smith, S., \& Lewis, S. D. (2011). Is Twitter a viable commercial use platform for small businesses? An empirical study targeting two audiences in the small business community. The Entrepreneurial Executive, 15, 73.

Giraldo Cardona, C. M., \& Martínez María-Dolores, S. M. (2017). Análisis de la actividad y presencia en facebook y otras redes sociales de los portales turísticos de las Comunidades Autónomas españolas. Cuadernos de Turismo, (39), 239-264. http://dx.doi.org/10.6018/ turismo.39.290521

González Fernández-Villavicencio, N. G. (2016). Bibliotecas, medios y métricas de la web social. In Anales de Documentación, 19(1), 1-13. http://dx.doi.org/10.6018/analesdoc.19.1.234001 
Gummerus, J., Liljander, V., Weman, E., \& Pihlström, M. (2012). Customer engagement in a Facebook brand community. Management Research Review, 35(9), 857-877. https://doi. org/10.1108/01409171211256578

Hudson, S., \& Thal, K. (2013). The impact of social media on the consumer decision process: Implications for tourism marketing. Journal of Travel \& Tourism Marketing, 30(1-2), 156160. https://doi.org/10.1080/10548408.2013.751276

Huertas, A., \& Marine-Roig, E. (2016). User reactions to destination brand contents in social media. Information Technology \& Tourism, 15(4), 291-315. https://doi.org/10.1007/s40558015-0045-9

Huertas, A., Setó-Pàmies, D., \& Míguez-González, M. I. (2015). Comunicación de destinos turísticos a través de los medios sociales. El profesional de la información, 24(1).

Jayasingh, S., \& Venkatesh, R. (2015). Customer engagement factors in facebook brand pages. Asian Social Science, 11(26), 19. http://dx.doi.org/10.5539/ass.v11n26p1

Kang, M., \& Schuett, M. A. (2013). Determinants of sharing travel experiences in social media. Journal of Travel \& Tourism Marketing, 30(1-2), 93-107. https://doi.org/10.1080/1054840 8.2013 .751237

Laroche, M., Habibi, M. R., \& Richard, M. O. (2013). To be or not to be in social media: How brand loyalty is affected by social media? International Journal of Information Management, 33(1), 76-82. https://doi.org/10.1016/j.ijinfomgt.2012.07.003

Leeflang, P. S., Verhoef, P. C., Dahlström, P., \& Freundt, T. (2014). Challenges and solutions for marketing in a digital era. European management journal, 32(1), 1-12. https://doi. org/10.1016/j.emj.2013.12.001

Márquez-Domínguez, C., López-López, P. C., \& Arias, T. E. (2017). Social networking and political agenda: Donald trump's Twitter accounts. In Information Systems and Technologies (CISTI), 2017 12th Iberian Conference on (1-6). IEEE.

Martínez-Fernández, V. A., Juanatey-Boga, Ó., Crespo-Pereira, V., \& Burneo, M. D. M. (2015). Prensa nativa dígital y redes sociales: cibermedios en Facebook y Twitter. International Journal of Information Systems and Software Engineering for Big Companies (IJISEBC), 2(2), 42-50.

Martínez, S. M, Bernal García, J. J., \& Mellinas, J. P. (2013). Análisis del nivel de presencia de los establecimientos hoteleros de la Región de Murcia en la Web 2.0. Cuadernos de Turismo, (31), 245-261.

Munar, A. M. (2012). Social media strategies and destination management. Scandinavian Journal of Hospitality and Tourism, 12(2), 101-120. https://doi.org/10.1080/15022250.2012.679047

Oviedo-García, M., Muñoz-Expósito, M., Castellanos-Verdugo, M., \& Sancho-Mejías, M. (2014). Metric proposal for customer engagement in Facebook. Journal of Research in Interactive Marketing, 8(4), 327-344. https://doi.org/10.1108/JRIM-05-2014-0028

Pathak, X., \& Pathak-Shelat, M. (2017). Sentiment analysis of virtual brand communities for effective tribal marketing. Journal of Research in Interactive Marketing, 11(1), 16-38. https://doi.org/10.1108/JRIM-09-2015-0069

Potgieter, L. M., \& Naidoo, R. (2017). Factors explaining user loyalty in a social media-based brand community. South African Journal of Information Management, 19(1), 1-9. https:// doi.org/10.4102/sajim.v19i1.744

Rosenthal, B., \& Brito, E. P. (2017). How virtual brand community traces may increase fan engagement in brand pages. Business Horizons, 60(3), 375-384. https://doi.org/10.1016/j. bushor.2017.01.009

Sánchez Casado, N., \& Giraldo Cardona, C. M. (2015). Análisis de la actividad en redes sociales de marcas del sector moda como herramienta de social commerce. Anuario de Jóvenes Investigadores, (8), 72-74.

Schmidt, M. (2014). Return on Investment ROI Explained: Definition, Meaning, and Example Calculations. Business Encyclopaedia. (https://goo.gl/XE1RyC) (22-07-2017) 
Sirakaya, E., \& Woodside, A. (2005). Building and testing theories of decision making by travelers. Tourism Management, 26(6), 815-832. https://doi.org/10.1016/j.tourman.2004.05.004

Sotiriadis, M. D., \& van Zyl, C. (2013). Electronic word-of-mouth and online reviews in tourism services: the use of twitter by tourists. Electronic Commerce Research, 13(1), 103-124. https://doi.org/10.1007/s10660-013-9108-1

Stockdale, R., Ahmed, A., \& Scheepers, H. (2012). Identifying Business Value From The Use Of Social Media: An Sme Perspective. In PACIS Proceedings, 169.

Taladriz-Mas, M. (2013). Los servicios de información y el retorno de la inversión: cómo llegar a conocerlo. El profesional de la información, 22(4).

World Travel and Tourism Cousin (WTTC, 2017). "Global economic impact and issues 2017". (https://goo.gl/mrPns4) (17-09- 2017).

Wozniak, T., Stangl, B., Schegg, R., \& Liebrich, A. (2017). The return on tourism organizations' social media investments: Preliminary evidence from Belgium, France, and Switzerland. Information Technology \& Tourism, 17(1), 75-100. https://doi.org/10.1007/s40558-0170077-4

Xiang, Z., \& Gretzel, U. (2010), Role of Social Media in Online Travel Information Search. Tourism Management, 31(2), 179-188. https://doi.org/10.1016/j.tourman.2009.02.016

Zeng, B., \& Gerritsen, R. (2014). What do we know about social media in tourism? A review. Tourism Management Perspectives, 10, 27-36. https://doi.org/10.1016/j.tmp.2014.01.001 